\title{
Differentially methylation of ANKRD53 and GATA3 genes in human miscarriages with trisomy 16
}

\author{
Ekaterina Tolmacheva \\ Research Institute of Medical Genetics, \\ TNRMC RAS, Tomsk, Russia \\ kate.tolmacheva@medgenetics.ru \\ Tatjana Nikitina \\ Research Institute of Medical Genetics, \\ TNRMC RAS, Tomsk, Russia \\ t.nikitina@medgenetics.ru \\ Ekaterina Serdyukova \\ National Research Tomsk State \\ University, Tomsk, Russia \\ katya.serdyukova.1997@mail.ru
}

\author{
Stanislav Vasilyev \\ Research Institute of Medical Genetics, \\ TNRMC RAS, Tomsk, Russia \\ stanislav.vasilyev@medgenetics.ru \\ Elena Sazhenova \\ Research Institute of Medical Genetics, \\ TNRMC RAS, Tomsk, Russia \\ elena.sazhenova@medgenetics.ru \\ Darja Zhigalina \\ Research Institute of Medical Genetics, \\ TNRMC RAS, Tomsk, Russia \\ dasha_150291@hotmail.com
}

Oksana Vasilyeva

Research Institute of Medical Genetics, TNRMC RAS, Tomsk, Russia oksana.vasilyeva@medgenetics.ru

Anton Markov

Research Institute of Medical Genetics, TNRMC RAS, Tomsk, Russia anton.markov@medgenetics.ru

Igor Lebedev

Research Institute of Medical Genetics, TNRMC RAS, Tomsk, Russia igor.lebedev@medgenetics.ru

\begin{abstract}
An excess dosage of the genes at the whole chromosome can lead to a disturbance of the epigenetic background of the entire genome. We analyzed the DNA methylation of several genes that are important for normal embryogenesis in miscarriages with aneuploidy and normal karyotype.
\end{abstract}

Keywords - DNA methylation, miscarriage, trisomy 16

Motivation and aim

\section{Motivation}

Trisomy of chromosome 16 is the most common aneuploidy among human miscarriages. Potentially, a supernumerary chromosome may affect the genome-wide methylation and disrupt the expression of genes necessary for normal embryo development. Previously, we analyzed the genome-wide methylation in chorionic villi in miscarriages with trisomy 16 using the Infinium HumanMethylation27 BeadChip (Illumina). A significant increase in DNA methylation was found for the 90 genes (deltaB $>0.15$ ) in miscarriages with trisomy 16 compared to induced abortions with normal karyotype.

Aim

We suggested that abnormal methylation in certain genes may be common for miscarriages with aneuploidy of various chromosomes.

\section{Methods}

Methylation of the individual gene promoters was studied by targeted bisulfite massive parallel sequencing in chorionic villi in 48 miscarriages with aneuploidy karyotype (26 with trisomy 16 and 22 with other autosomal trisomies and monosomy of the $\mathrm{X}$ chromosome), 8 miscarriages with normal karyotype and 7 induced abortions.

\section{Results}

We assessed the methylation level in promoters of two top differentially methylated genes (deltaB $>0.20)$ - ANKRD53 and GATA3. Methylation of the CpG sites of these genes did not differ in miscarriages with normal karyotype compared with induced abortions. Miscarriages with trisomy 16, but not miscarriages with other aneuploidies, had higher levels of DNA methylation in ANKRD53 (for 5 from 40 analyzed CpG-sites, $\mathrm{p}<0.05$ ) and GATA3 (for 49 from 171 analyzed CpG-sites, $\mathrm{p}<0.05)$ genes. GATA3 gene encodes transcription factor, which is a key regulator of trophoblast differentiation, and imbalance of its expression can lead to aberrant trophoblast invasion. ANKRD53 is involved in spindle dynamics and nucleus integrity in mitosis. Thus, abnormal expression of these genes can dramatically affect embryonic development and lead to mosaic karyotypes that are often observed in cases of trisomy 16.

\section{ACKNOWLEDGMENT}

The study was supported by the Russian Science Foundation (19-74-10026). 